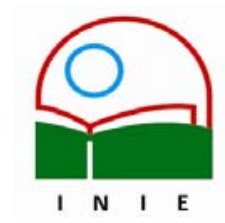

Universidad de Costa Rica

Facultad de Educación

Instituto de Investigación en Educación

ACTUALIDADES INVESTIGATIVAS EN EDUCACION

\title{
INCORPORACIÓN DE LAS TECNOLOGÍAS DE INFORMACIÓN Y COMUNICACIÓN EN LA DOCENCIA UNIVERSITARIA ESTATAL COSTARRICENSE: PROBLEMAS Y SOLUCIONES
}

\begin{abstract}
Johel Brown Grant ${ }^{1}$
Resumen: Este artículo presenta un análisis de la situación actual de la incorporación de las Tecnologías de Información y Comunicación (TIC) en la docencia universitaria estatal costarricense. La primera parte discute los problemas que dificultan los procesos de incorporación de estas tecnologías. En la siguiente sección se propone un conjunto de soluciones que puedan servir de base para iniciar estos procesos o mejorarlos en los casos donde ya se hayan iniciado. La reflexión concluye con el argumento de que la solución final de la problemática de la incorporación de las TIC en la docencia depende, entre otros aspectos, de un cambio de actitud de las autoridades de las universidades estatales y la creación de mayores y más eficaces espacios para la discusión de estos temas.
\end{abstract}

Palabras clave: INCORPORACIÓN DE TECNOLOGÍAS DE INFORMACIÓN Y COMUNICACIÓN, DOCENCIA UNIVERSITARIA, TECNOLOGÍA EDUCATIVA.

Abstract: This article analyzes the issues that currently hinder the efforts to integrate Information and Communication Technologies (ICT) into instruction in the Costa Rican state-run higher education system. To address these issues, the article offers a set of solutions that may be used as a basis to improve ongoing ICT integration efforts or as a springboard for ICT integration initiatives. As a conclusion the article poses that the ultimate solution for ICT integration into college teaching involves a change of attitude among university authorities and the opening of wider and more effective discursive spaces to flesh out these issues.

Key words: INFORMATION AND COMMUNICATION TECHNOLOGY INTEGRATION, UNIVERSITY TEACHING, EDUCATIONAL TECHNOLOGY.

\section{Introducción}

La discusión de la formación pedagógica de los docentes universitarios no puede ser completa si no se consideran aspectos que expliquen la relación que hay entre los profesores, la tecnología, el aula y los alumnos. Si bien es cierto que la formación pedagógica de los docentes ha tenido que incluir algunos aspectos de la tecnología a lo largo de la historia, la discusión de este tema cobra una importancia especial en este momento por la forma como las nuevas Tecnologías de Información y Comunicación (TIC) han modificado las sociedades occidentales.

\footnotetext{
1 Doctor en Comunicación y Retórica con especialidades en Comunicación Mediante Computadores e Interacción Humano Computadora por el Instituto Politécnico Rensselaer, EEUU. Consultor y profesor de tecnología educativa para empresas de software y universidades nacionales y extrajeras. Ex director del Campus Virtual UCR Interactiva de la Universidad de Costa Rica; actualmente es profesor de la Facultad de Educación de la misma universidad y sus áreas de investigación se concentran en el diseño de la experiencia del usuario en ambientes digitales y los procesos de integración de la tecnología en la docencia universitaria.
}

Correo electrónico 1johel@gmail.com

Artículo recibido: 6 de mayo, 2005

Aprobado: 20 de junio, 2005 
Aunque existe un consenso acerca de la necesidad de incluir más temas relacionados con el uso de las TIC en la formación pedagógica de los docentes, los esfuerzos en este sentido no se han dado de acuerdo con políticas formales o de acuerdo con modelos que hayan surgido de un período de reflexión y análisis. De hecho existen muchos factores que han creado obstáculos para la incorporación eficaz de las TIC en la docencia. Por eso, ante los cambios vertiginosos de las $\mathrm{TIC}$, el reto de las universidades es ofrecer herramientas y estrategias que permitan una mejor incorporación y aplicación de estas a la docencia. Esta reflexión analiza los principales problemas que dificultan la integración de estas tecnologías en la docencia universitaria estatal costarricense, y discute algunas posibles soluciones que puedan ser parte de un modelo de formación pedagógica que incluya formación en la incorporación de las TIC en la docencia.

\section{La problemática}

Los principales obstáculos para la incorporación efectiva de las TIC en la docencia son más evidentes en las áreas de la capacitación técnica y didáctica de los docentes en el uso de las TIC, en sus actitudes hacia las ellas, en la falta de motivación y en la forma cómo las autoridades académicas o administrativas introducen las nuevas TIC en el ambiente universitario. Los problemas en estas áreas se discuten a continuación.

\subsection{Capacitación didáctica y técnica}

Cuando se habla de la integración de las nuevas TIC en la docencia universitaria hay que distinguir dos tipos de capacitación: la capacitación didáctica que se ocupa de ayudar al docente a encontrar estrategias para integrar las TIC en la docencia de forma efectiva y provechosa, y la capacitación técnica que ayuda al docente a desarrollar las habilidades necesarias para usar esas tecnologías.

\subsubsection{Capacitación didáctica}

Según Green (2002), la capacitación en las diferentes metodologías y estrategias de incorporación de las TIC en la docencia es la preocupación más importante de los docentes. En este sentido, los docentes se muestran preocupados por obtener una capacitación que les permita utilizar las TIC de acuerdo con criterios didácticos más eficientes que posibiliten un aprovechamiento efectivo del tiempo dentro y fuera del aula, que estimulen el aprendizaje autónomo y ofrezcan experiencias educativas significativas. El principal obstáculo para la consecución de estas metas es que las universidades estatales no ofrecen espacios 
formales, como centros de tecnología educativa, donde los docentes puedan acudir para obtener capacitaciones de esta naturaleza. Más aún, existe un déficit de recursos humanos preparados, y con suficiente experiencia, para capacitar a los docentes en la incorporación efectiva de las TIC (Pasquinelli, 1998), pues tal capacitación, como indican Van Dusen (1998) y Cuban (2001), no es un aspecto prioritario en los programas de formación pedagógica. Por lo general en estos programas de formación el personal trabaja con los docentes en actividades de inducción a la docencia universitaria, diseño curricular, selección de estrategias didácticas para el desarrollo y la presentación de los materiales y contenidos de los cursos, y otras actividades propias de la formación pedagógica de los docentes. Sin embargo, la incorporación de las TIC no necesariamente figura en los objetivos de los programas de formación pedagógica de los docentes, ya que es probable que el personal que capacite pedagógicamente a los docentes no vea como una prioridad el prepararse para capacitar a los docentes en el uso didáctico, la selección y evaluación de las TIC; ni considere necesario prepararlos para asumir los nuevos roles que surgen al incorporar TIC en la docencia. La falta de estos conocimientos, así como de otros requisitos de perfil profesional, hace aún más difícil el nombramiento de personal calificado para proporcionar este tipo de capacitaciones. En este sentido solo existen en el país dos programas de posgrado en tecnología educativa y sus programas curriculares no incluyen cursos orientados a la incorporación de las TIC en la educación superior ni cursos que ofrezcan metodologías para la capacitación de docentes en el uso pedagógico de las TIC.

\subsubsection{Capacitación técnica}

Aunque las universidades estatales costarricenses poseen medios y equipos audiovisuales para el desarrollo de las actividades docentes el personal de apoyo que conoce de su funcionamiento no necesariamente está preparado para capacitar a los profesores en el uso de los mismos. Este un fenómeno común en muchas universidades (Pasquinelli, 1998). En estos casos las funciones más comunes del personal técnico son las de proveer a los docentes los recursos audiovisuales, mecánicos o informáticos que éstos necesitan; por lo tanto, aunque este personal tuviera alguna preparación para capacitar a los docentes, esta actividad no necesariamente está contemplada como parte de sus funciones esenciales.

Para que sea efectiva, la capacitación técnica tiene que darse de acuerdo con ciertos objetivos didácticos. En otras palabras, esta capacitación debe ofrecer al usuario un conocimiento general del objeto, pero dentro de un contexto en el cual el usuario pueda apreciar las funcionalidades del mismo a la luz de sus aplicaciones didácticas. La meta de la 
capacitación técnica debe ser de ayudar al usuario a crear modelos mentales de la funcionalidad del objeto en un contexto de uso didáctico.

\subsubsection{Apoyo técnico}

En este caso los problemas más comunes surgen porque el personal de apoyo técnico tiene horarios limitados; es insuficiente para atender la demanda o, en algunos casos, no es el idóneo pues, aunque instala los equipos, no necesariamente puede repararlos o solucionar complejos problemas técnicos que éstos presenten. Otro problema que se puede citar es que el personal de apoyo técnico no posee suficiente preparación en los métodos más efectivos para dar ayuda a los usuarios, ya sea en forma presencial, por teléfono o en línea. Además es importante mencionar la falta de apoyo técnico en el uso de las TIC para la producción de materiales educativos. Existe una escasez de personal permanente que ayude a los profesores a producir, por ejemplo, videos educativos, simulaciones, videoconferencias o pequeños programas de software para demostraciones, prácticas, encuestas o evaluaciones en línea.

\subsubsection{Educación continua}

Los problemas anteriormente descritos surgen, principalmente, porque las universidades estatales costarricenses no cuentan con centros dedicados exclusivamente a la capacitación continua de los docentes en el uso y la integración de las TIC. Sin embargo cada vez hay más presión para que los docentes incorporen las TIC en la docencia. De ahí la necesidad de crear espacios físicos e institucionales que ofrezcan este entrenamiento, no solo por los cambios constantes en la tecnología, sino porque las universidades tienen un flujo continuo de nuevos docentes que, aunque estén familiarizados con las TIC, necesitan capacitarse en su uso didáctico

Por otro lado, aun no existen espacios de conversación para que los profesores puedan compartir experiencias sobre la integración de las TIC. En este caso, las buenas prácticas o los esfuerzos exitosos no se conocen porque no existen canales de comunicación formal o informal entre diferentes escuelas, facultades o, inclusive, universidades. El intercambio de experiencias que surgiría de estos espacios de conversación sería de gran ayuda para la formación continua de los profesores y serviría como una fuente de estrategias y recursos nuevos.

La educación continua en la incorporación de las TIC en la docencia es, por lo tanto, necesaria pues ayuda a los docentes a obtener un dominio más sofisticado de la tecnología 
y sus aplicaciones didácticas, al transformar su condición de usuarios novatos a la de usuarios intermedios y avanzados.

\subsection{Actitud hacia la tecnología}

Otro asunto que complica aún más la discusión es la actitud de los profesores ante la tecnología. Es muy común encontrar docentes que miran con escepticismo, resistencia o abierta hostilidad, la incorporación y el uso de las TIC en la docencia (Salinas, 2002) pues sienten que esta integración disminuiría su autoridad o impacto en el aula. También se citan como razones para este escepticismo la falta de evidencias de la efectividad de las TIC, la inhabilidad de los estudiantes para desarrollar el pensamiento crítico como resultado de su interacción con TIC y, por parte de los docentes, su falta de tiempo para la capacitación o su falta de conocimiento pleno de estas tecnologías (Escamez y Martínez, 1987). Estas actitudes negativas también se basan en la idea de que, para muchos docentes, la incorporación de las TIC pondría en evidencia la brecha digital que existe entre ellos y sus alumnos; esto significaría una pérdida de credibilidad en el aula para los docentes. Además tendrían que considerar seriamente hacer cambios en la metodología de enseñanza para ajustarla a un proceso de interacción más igualitario y más flexible (Salinas, 2002).

Muchos docentes adoptan esta actitud negativa porque sienten que de alguna forma $u$ otra la tecnología va a suplantarlos o hacerlos menos efectivos como docentes. Este argumento se remonta a mediados del siglo XX cuando el prometido impacto en la educación que tendrían tecnologías como la televisión y la computadora no se dio (Schramm, 1977) porque se trató de utilizarlas como sustitutos de los docentes. En el caso de la televisión, los docentes aparecían en pantallas presentando el contenido del curso y los estudiantes debían recibir la información de la televisión tal como si tuviesen al docente en el aula.

Este tipo de argumentos es la fuente del escepticismo de muchos docentes que, más que considerar a las TIC como un complemento de la enseñanza y un instrumento para enriquecer el aprendizaje, las visualizan como un elemento de distracción en sus cursos. Asimismo, el escepticismo también está relacionado con investigaciones que sugieren que el impacto positivo de la incorporación de las TIC en la educación ha sido poco (Ehrman, 1995), lo cual ha contribuido a la percepción de que las TIC no tienen mucho que ofrecer mas allá de la novedad.

Como se mencionó, algunas de las actitudes negativas se basan en la percepción de que las TIC, especialmente las computadoras, no son capaces de estimular el pensamiento crítico, esto con base en la tradicional visión de de las TIC como herramientas para almacenar y 
difundir contenidos. Este concepto se enmarca dentro de la idea de que los estudiantes aprenden de las computadoras, en lugar de aprender con ellas (Jonassen, et. al., 1998) y está íntimamente relacionada con la opinión de muchos docentes de que el propósito de las computadoras y otras TIC es de suplantarlos en el aula.

Para entender porqué se desconfía de las TIC en el ámbito del pensamiento crítico es necesario considerar dos aspectos importantes. Primero, existe la corriente que postula que el pensamiento crítico no se puede enseñar, sino que este debe desarrollarse de manera intrínseca en cursos que obliguen a los estudiantes a desarrollar sus habilidades cognitivas superiores-comprensión, aplicación, síntesis, integración y evacuación. Entonces, considerando el argumento de las TIC como herramientas con las que se enseña contenido, éstas ofrecerían muy poco en el desarrollo de habilidades cognitivas que normalmente surgen de la interacción entre el profesor, los alumnos y las lecturas de los textos del curso. Por otra parte existe una corriente que propone que el pensamiento crítico puede enseñarse en cursos como lógica o pensamiento critico con resultados modestos (Van Gelder, 2000). Sin embargo, en este sentido, el escepticismo de muchos docentes que critican esta posición se basa en la dificultad de demostrar cómo las TIC, en especial la computadora, pueden ayudar al estudiante a transferir, de un dominio a otro, habilidades cognitivas como la síntesis o la aplicación (Van Gelder) cuando se les enseña pensamiento crítico. En esta corriente, tanto como en la anterior, se considera que TIC como la computadora son herramientas de las que se aprende contenido, son herramientas eficaces para aprender a manipular software $\mathrm{u}$ otras aplicaciones tecnológicas, pero no son herramientas para desarrollar las habilidades cognitivas superiores.

\subsection{La falta de motivación}

La incorporación de las TIC en la docencia se ve afectada, en muchos casos, por la falta de motivación de los profesores. Varios estudios (Schifter, 2000; Rogers, 2000, Rockwell, Schauer, Fritz y Marx, 1999; Rao \& Rao, 1999) sugieren que las razones que explican este fenómeno son, por lo general, problemas relacionados con las condiciones de acceso a la tecnología o la falta de acceso a la misma; la existencia de tecnología atrasada o inservible; la falta de capacitación en la tecnología actualizada; la falta de apoyo técnico, y problemas de conectividad o de velocidad de descarga, entre otros.

Sin embargo, uno de los aspectos más desmotivantes es la falta de tiempo (Lee, 2001) para la dedicación a la capacitación y la formación continua. Esta es la queja constante de muchos docentes. Desafortunadamente no son muchas las universidades que liberan a los 
profesores de algunas de sus cargas académicas o administrativas para que empleen este tiempo para complementar su formación pedagógica con capacitación en el uso y la incorporación de las TIC. Los casos donde los profesores logran esta capacitación o formación continua casi siempre son el resultado de su propia iniciativa, la cual casi nunca se recompensa.

Otro factor que influye en la falta de motivación es la alta inversión de tiempo que requiere la incorporación de las TIC en la docencia. Según Adams (2002), Kagima y Hausafu (2001) y Leggert y Persichitte (1998) la falta de tiempo crea barreras, tanto para desarrollar actividades de exploración, capacitación y práctica con las TIC, como para incorporarlas en la preparación de cursos y materiales. El docente, entonces, debe medir su tiempo personal con la cantidad de tiempo que le ofrece la universidad para dedicarse a los procesos de incorporación de las TIC; en alguna medida estás valoraciones incidirán en su nivel de motivación y su decisión final.

\subsection{Estrategias de incorporación de las TIC en las universidades}

Los factores que contribuyen a la problemática de la incorporación y que inciden fuertemente en el interés y la motivación del profesorado son las estrategias utilizadas para incorporar las TIC en las universidades. Varios autores apuntan que la aceptación de la tecnología está determinada por qué tan útil la encuentran los usuarios y su percepción de qué tan fácil es para otros usuarios (Rogers, 1986; Trevino, Lengel, y Daft, 1987). Por esta razón la forma como la nueva tecnología se inserta en el medio es tan importante como la tecnología misma. Por ejemplo, al introducir las nuevas TIC no siempre se hace énfasis en lo fácil que es usarlas (porque muchas veces no lo es). Sin embargo, información de este tipo es importante para generar interés en la adopción de la nueva tecnología. Además, la presentación de la nueva tecnología no siempre está acompañada por procesos de seguimiento o de recursos de entrenamiento y refrescamiento permanente. Es así como, en ocasiones, se presentan cursos iniciales para usar la nueva tecnología, pero al final de cierto período los capacitadores se van y los usuarios quedan sin recursos para consultas o ayudas.

Cuando se presenta la nueva tecnología los usuarios desarrollan expectativas como accesibilidad, facilidad de uso, aplicación a su entorno diario, tiempo razonable para ejecución de las principales tareas, entre otras. Sin embargo, no siempre se satisfacen estas expectativas, porque en la presentación de la tecnología se enfatiza lo que ésta puede hacer, sus nuevos elementos, sus nuevas virtudes, etc. y se le da poca o ninguna importancia a la 
experiencia de los usuarios interactuando con la tecnología. Es precisamente en este punto donde residen las preguntas y las expectativas de los docentes; es por eso que su motivación e interés, en muchos casos, estará íntimamente ligada a cómo se contestan esas preguntas y a cómo se llenen esas expectativas.

Esta situación es aún más compleja porque, en ocasiones, la adquisición de la tecnología responde a intereses políticos y económicos que no necesariamente ponen la experiencia del usuario en un plano principal, pues los grupos encargados de adquirir la tecnología o tomar decisiones sobre su adquisición no están sensibilizados a la necesidad de optimizar la experiencia de los usuarios con la tecnología. En otros casos la adquisición de la tecnología no se hace de acuerdo con un plan estratégico de desarrollo tecnológico en la universidad, o de acuerdo con un plan que claramente demuestre el impacto que tendría en la población meta, desde el punto de vista de su experiencia como potenciales usuarios de la tecnología que se va a adquirir. Estos escenarios son más comunes en sectores universitarios que desean incorporar las TIC sin haber determinado cómo estas enriquecerían el aprendizaje o la práctica docente.

Otro aspecto problemático de las estrategias de inserción de las TIC es que los mecanismos utilizados para comunicar a la comunidad universitaria sobre la adquisición e incorporación de las TIC no siempre tienen un gran alcance. Es común que, aunque la tecnología se inserta en varios polos importantes de la institución, la información útil acerca de la misma solo llega a algunos de los sectores que están más cerca de aquellos que gestaron la adquisición, inserción o puesta en marcha de la tecnología.

\section{Soluciones}

La discusión anterior demuestra que la problemática de la incorporación de las TIC es compleja pues abarca múltiples dimensiones que interrelacionan la cultura tecnológica existente en las universidades, las decisiones que toman las autoridades universitarias, las acciones de los docentes y el contexto físico y tecnológico en el cual interactúan estas variables. Esto significa que las soluciones que se propongan deben satisfacer las necesidades que imponen las diferentes variables presentes. Es por esto que no es posible ofrecer una única solución para esta problemática, sino un conjunto de soluciones en las cuales diferentes sectores de la universidad contribuyan a los procesos de incorporación de las TIC. En este sentido el modelo de innovación que presenta Fullan (1994) puede ser de gran utilidad. En este modelo Fullan señala que las estrategias "arriba-abajo", o "abajo- 
arriba", para la innovación tecnológica, no funcionan por sí mismas. Según el autor, lo que se requiere es de una combinación adecuada de ambas.

Tomando como base este modelo, podemos identificar entonces dos áreas donde se pueden implementar cambios que contribuyan a la solución de la problemática de la incorporación de las TIC en la docencia universitaria estatal costarricense. Estas dos áreas son el área institucional-administrativa y el área docente. La estrategia que aquí se sugiere propone cambios a nivel institucional que afecten al cuerpo docente (desde arriba hacia abajo) y cambios en el área docente que tengan una influencia en el sector institucional administrativo (desde abajo hacia arriba).

Una estrategia de soluciones combinada, como la que aquí se propone, sería de gran beneficio, porque la influencia que ejercería un grupo sobre el otro tendría un efecto positivo en el aula, el laboratorio, y demás espacios donde el proceso de enseñaza y aprendizaje sería enriquecido con la incorporación de las TIC.

A continuación se describen las acciones que se deben implementar en el nivel institucional.

\subsection{El nivel institucional administrativo}

Existe un consenso de que las estrategias de incorporación de las TIC en la docencia universitaria requieren del apoyo institucional (Salinas, 2004; Rogers, 2000; Hofffman, 1996; Sherry, 1998; y Stockdill y Morehouse, 1992, entre otros) para lograr una mayor efectividad y alcance en su aplicación. En este sentido, el apoyo institucional debe traducirse en acciones concretas y sistemáticas para solucionar la problemática planteada Estas acciones se resumen en la siguiente lista:

- Articulación de una política institucional de innovación

- Impulso a la educación continua

- Mejora de la comunicación interinstitucional

- Desarrollo de un plan de Incentivos

- Mejora de la logística e infraestructura

\subsubsection{La articulación de una política institucional de innovación}

La problemática aquí planteada se debe, en parte, a la falta de una clara política de innovación tecnológica. Esto exige que, como un primer paso para implementar soluciones, las universidades estatales costarricenses articulen políticas de innovación tecnológica concretas y bien fundamentadas ya que "la ausencia de un sólido plan estratégico limita severamente la adopción y uso generalizado de la tecnología en la educación" (Epper, 2004, 
p. 22). La articulación de esta visión implica la creación de instancias institucionales dedicadas a detectar y señalar dificultades para incorporar las TIC; evaluar planes y programas cuyo fin es lograr esa incorporación y, finalmente, hacer recomendaciones para la innovación tecnológica. A estas estrategias hay que agregar el desarrollo de un plan estratégico para el presupuesto de la tecnología (Epper). Este presupuesto no estaría dedicado únicamente a la adquisición de equipo e instrumentos, sino a la adquisición de personal técnico, de capacitadores y personal de apoyo en la producción de materiales y otros recursos educativos.

Esta visión también implica la creación y difusión de documentación e información de estas políticas para articular un discurso universitario sobre la incorporación de las TIC. No obstante, hay que destacar que tal visión y tal discurso deben ser el resultado de una discusión amplia y participativa entre todos los sectores universitarios.

La creación de una visión institucional implica, entonces, un cambio en la forma tradicional como las universidades estatales vislumbran la integración de las TIC, porque requiere que estas se desprendan de la tradicional rigidez burocrática que las caracteriza. Asimismo, esta política institucional implica un compromiso sostenido con la comunidad universitaria y es el primer paso en el desarrollo de una cultura de integración de las TIC en la docencia universitaria.

\subsubsection{Impulso a la educación continua}

Para que la política de innovación que instituya la universidad sea exitosa requiere del apoyo docente, ya que este grupo también debe ejecutar, en alguna medida, las acciones que requiere la implementación de una política institucional y, más aún, es este grupo el que debe enfrentar los resultados de esas acciones. De hecho, el convencimiento de los profesores, junto al apoyo institucional, son la clave del éxito para la integración de las TIC en la docencia universitaria (Salinas, 2004).

La obtención del apoyo se logra, fundamentalmente, estableciendo recursos y programas que eduquen y capaciten a los docentes en el uso y la aplicación didáctica de las TIC.

A continuación se describen las estrategias que proveerían una solución en esas áreas:

\subsubsection{Capacitación de los profesores}

Un programa de capacitación efectivo incluiría las cuatro áreas fundamentales del proceso de la instrucción: el instructor, el alumno, el contexto educativo y los materiales educativos. 
A nivel del instructor la capacitación proporcionaría al docente estrategias específicas para integrar las TIC en el aula y adaptarlas a sus necesidades curriculares ya que, como indica Bates (2001), sin cambios en los métodos de enseñanza, el uso de la tecnología no es más que una sobrecarga para los profesores. Por otro lado, la capacitación prepararía a los docentes para enfrenar los cambios que surgen de este proceso de incorporación. Uno de estos cambios sería la adopción de un modelo de instrucción que enfatice el aprendizaje flexible; este modelo requiere que el docente transforme su rol de autoridad y fuente primaria de conocimientos en el aula, al rol de guía y mentor del alumno y de su proceso de aprendizaje (Latona, 1996, Salinas, 1997, Moran y Myringer, 2000). Este cambio es importante porque la educación enfrenta un proceso en donde las TIC permiten a los estudiantes mayor autonomía e independencia en su aprendizaje.

Además, para que el rol facilitador del profesor sea más efectivo, éste debe preparar al alumno para asumir un rol más activo, autónomo e independiente en su aprendizaje, lo cual en sí es una consecuencia natural de la integración de las TIC en aula. Además, debe preparar al estudiante para enfrentar la nueva tecnología y las exigencias que esto implica para su experiencia ya que los cambios que introducen las TIC afectan su contexto educativo (Salinas, 1997).

A nivel del contexto educativo, la capacitación permitiría a los docentes aprender a usar las TIC, actualizarse en ellas, enfrentar sus limitaciones y desarrollar criterios para seleccionarlas y evaluarlas de forma tal que causen el impacto más efectivo en el proceso educativo.

En el área de los materiales educativos, la capacitación podría incluir ideas o consejos útiles para utilizar las TIC en el desarrollo de estos materiales; también se incluirían estrategias para mejorar la presentación de materiales desarrollados con las TIC. El punto medular de esta capacitación sería la discusión de estrategias y metodologías que permitan la utilización de las TIC para crear materiales y recursos que ayuden a enriquecer la docencia y potenciar el aprendizaje de los estudiantes a través de experiencias educativas memorables y significativas.

\subsubsection{Capacitación de personal técnico}

Como complemento de la capacitación docente, las universidades estatales deben invertir en la capacitación de personal técnico que pueda servir de soporte a los docentes en la preparación de material educativo y que les pueda capacitar en la manipulación de las herramientas e interfaces de las TIC. Las universidades que hacen inversiones estratégicas 
en la innovación tecnológica constantemente reclutan a personal técnico y lo capacitan para ofrecer apoyo y consultoría técnica al personal docente.

\subsubsection{Mejora de la comunicación institucional}

La acción principal de cualquier proceso institucional de incorporación de las TIC debe ser la de fortalecer la comunicación que existe entre los diversos grupos de interés universitarios. El tipo de comunicación que redunda en esfuerzos de integración exitosos se basa en el diálogo entre las partes interesadas. En este caso los administrativos deben ser sensibles a las necesidades que tienen los docentes y estos deben, por otro lado, hacérselas saber a la administración. Como elemento mediador de esta conversación figuraría una política institucional que claramente delimite las condiciones en que se puede dar ese discurso. Esto contribuiría a que la comunicación entre estos sectores fuera más provechosa y efectiva.

Otro aspecto que mejora la comunicación interinstitucional es la creación de espacios donde los docentes puedan discutir o intercambiar experiencias relacionadas con la integración de las TIC, y donde puedan aprender de la experiencia de otros docentes y generar ideas que les ayuden a encontrar estrategias de incorporación efectivas.

Estos espacios pueden ser foros informales organizados en las unidades académicas como oportunidades para que el personal docente comparta su conocimientos con otros. Además, las universidades pueden crear espacios más formales como coloquios, conferencias o simposios interuniversitarios e, inclusive, centros de capacitación en el uso didáctico de las TIC, donde se pueda desarrollar el discurso interdocente en estos temas. Lo importante de la existencia de estos espacios es que permite el flujo de información entre los docentes y también favorece la gesta de argumentos para la conversación institucional que surge entre ellos y los administrativos. Estas experiencias constituyen una forma de educación continua para los docentes pues les permite reflexionar sobre su experiencia en los procesos de integración de las TIC.

El fortalecimiento de la comunicación interinstitucional se basa en la capacidad que tenga la institución de difundir información sobre las innovaciones, las capacitaciones necesarias para esas innovaciones y, en algunos casos, información logística como la ubicación física de las innovaciones. Esta capacidad implica no solo la existencia de las herramientas de difusión (excelentes en muchas universidades estatales costarricenses), sino la utilización efectiva de estas herramientas para difundir la información al público meta en el momento propicio. Esta capacidad también implica la habilidad de las universidades de emitir información clara y específica sobre asuntos relacionados con la innovación y la integración de las TIC. La 
difusión de la información, por ende, no puede limitarse a la publicación de noticias sobre la adquisición de nuevo equipo; también debe incluir información que permita conocer, utilizar, e integrar esa innovación.

La capacidad de difusión también implica la habilidad de transmitir de mensajes que sean lo suficientemente persuasivos y educativos para convencer a los docentes (muchos de los cuales son por lo general escépticos hacia las TIC) para que incorporaren las TIC en la docencia.

Un argumento adicional que se debe considerar para mejorar la comunicación interinstitucional es la comunicación hacia los estudiantes. En este caso los docentes necesitan capacitación para modificar sus estrategias de comunicación con los estudiantes en lo referente al uso de las TIC. Si bien es cierto que los estudiantes generalmente tienen una disposición positiva a las TIC, su experiencia del uso de las TIC en el aula debe ser mediada por la intervención docente. En este caso el docente debe preocuparse por cómo introduce la tecnología en el aula; cómo retroalimenta a sus estudiantes en el uso de esta tecnología y cómo comunica a los estudiantes acerca los espacios donde pueden encontrar ayuda, si surgen problemas relacionados con la tecnología. Esto implica un buen conocimiento de las necesidades tecnológico-educativas de los estudiantes.

\subsubsection{Desarrollo de un plan de incentivos}

Otra de las acciones que contribuirían de forma positiva a la solución de la problemática es el establecimiento de un plan de incentivos que premien los esfuerzos de los docentes. La justificación fundamental de un plan de incentivos para la incorporación de las TIC se basa en que la responsabilidad y las cargas adicionales son mayores para los docentes que incorporan las TIC, que para los que no lo hacen. La cantidad de horas dedicadas a la preparación de material de clase, la comunicación con los estudiantes y la capacitación para el uso y la aplicación de la TIC, entre otros aspectos, pueden hacer de la docencia universitaria mediada por las TIC una labor titánica.

Quienes critican la implementación de un plan de incentivos, consideran que la labor adicional que realizan los docentes para incorporar las TIC es parte normal del trabajo académico y no comparan sus esfuerzos con los de aquellos docentes cuyos intereses están enfocados en otros objetivos.

Es así cómo muchos docentes no se preocupan por invertir su tiempo en realizar actividades tendientes a incorporar las TIC en la docencia, ya que no existe un reconocimiento a estos esfuerzos. Un plan de incentivos es importante porque no solo reconoce la labor que realiza 
el docente en este campo, sino que se constituye en una fuente de estímulo hacia el esfuerzo que hacen los docentes por incorporar las TIC en la docencia.

\subsubsection{Características de un plan de incentivos}

Un plan de incentivos para los docentes debe ser parte integral de una estrategia de inversión en las TIC. Aunque cada institución debe decidir cómo estructurar este plan, es necesario señalar algunos aspectos que deberían ser parte integral de un programa de incentivos para los docentes que realicen esfuerzos en esta línea.

\subsubsection{Incentivos académicos}

En una encuesta realizada entre profesores universitarios Schifter (2000) encontró que la obtención de puntos para el ascenso en el régimen académico es un factor que motivaría fuertemente a los a docentes a trabajar en un ambiente de aprendizaje asincrónico mediado por la tecnología.

Este ejemplo ilustra la importancia de los incentivos académicos en el desarrollo de estrategias de incorporación de las TIC. Estos incentivos académicos deben incluir puntajes para el ascenso en el régimen académico, certificados de participación o conclusión de capacitaciones, entre otros reconocimientos.

\subsubsection{Incentivos económico-laborales}

La falta de compensación salarial es una de los obstáculos para la incorporación de las TIC en la docencia universitaria (Rao \& Rao, 1999). Esto es comprensible si se evalúan los costos que implican la preparación y capacitación para estos procesos; ya que estos costos, por lo general, son cubiertos por los docentes.

En un plan de incentivos la compensación salarial figuraría como reconocimiento al mérito (Shifter) y al esfuerzo adicional de los docentes. Sin embargo, otros importantes incentivos laborales son la liberación de cargas administrativas o académicas para asistir a las capacitaciones y la dotación de personal de apoyo para el empleo y aplicación de las TIC. En algunos casos un estímulo adicional puede estar relacionado con la reducción en la cantidad de estudiantes matriculados en cursos donde los docentes incorporen las TIC.

\subsubsection{Incentivos tecnológicos}

Entre los incentivos tecnológicos más importantes se encuentra el fácil acceso a los recursos tecnológicos, la actualización y buen funcionamiento de los equipos, su facilidad de Volumen 5, Número 1, Año 200514 
aprendizaje y de uso y, en algunos casos, la participación de los docentes en la toma de decisiones relacionadas con la adquisición de los recursos.

\subsubsection{Mejora de la logística e infraestructura}

La credibilidad del apoyo institucional a los esfuerzos de incorporación de las TIC está basada, en gran medida, en los cambios y las modificaciones logísticas que realice la universidad. Por ende, el apoyo institucional se debe reflejar en la actualización constante del equipo, pues muchos docentes pierden interés en incorporar las TIC cuando los recursos que les ofrece la universidad están desactualizados o muy obsoletos para interactuar con la nueva tecnología. Las siguientes observaciones ofrecen algunos aspectos de logística e infraestructura que se deben considerar como parte de un conjunto de soluciones a la problemática que se ha presentado.

\subsubsection{Facilidades de acceso al equipo}

En algunos casos será necesario que las universidades estatales modifiquen los procesos burocráticos que dificultan el acceso de los docentes a equipos especializados. Esto implicará, en algunos, casos cambios en los horarios de préstamo de recursos o la reducción del papeleo necesario para el préstamo de los equipos.

Otro aspecto donde se deben introducir cambios es en los procesos de licitación para la adquisición del equipo en las universidades estatales. Estos trámites pueden demorar la compra del equipo al punto de que éste sea ya obsoleto, cuando finalmente es adquirido, ya que durante el período de adquisición, habrían surgido nuevas necesidades tecnológicas no contempladas en el cartel de licitación original.

\subsubsection{Tecnología centrada en los usuarios}

Es difícil comprobar cuánto median los criterios de usabilidad en la selección de las TIC para su incorporación en la docencia, ya que, en muchos casos, los criterios económicos son los que tienen mayor peso en la selección final. Sin embargo este es un tema que debe discutirse, pues lo docentes necesitan utilizar TIC que tengan interfases intuitivas, claras y de fácil aprendizaje y uso. El éxito de la incorporación de las TIC en la docencia estará determinado, en gran medida, por la existencia de estas condiciones.

Como parte de los esfuerzos de integración es necesario que las instituciones educativas tengan estándares mínimos de usabilidad y ergonomía que rijan la selección de las TIC. Estos estándares deben formar parte de un plan estratégico de inversión en la tecnología Volumen 5, Número 1, Año 200515 
pues solo así será posible discutir aspectos como el impacto que tendrán las TIC seleccionadas en la experiencia de los usuarios. La existencia de estos estándares mínimos forzarán a las autoridades a cuestionar qué tan fáciles de aprender y utilizar son las TIC; qué mecanismos de ayuda son necesarios y qué tan efectivos son y, en algunos casos, cuál ha sido la experiencia de usuarios similares.

\subsubsection{Participación docente en los procesos de selección de la tecnología}

Es necesario que se incluya a docentes que representen a los usuarios meta en el proceso de selección de las TIC. Cuando la selección de las TIC se deja solo a grupos en la esfera administrativa o grupos entre los que no haya usuarios meta hay una mayor riesgo de que la tecnología adquirida no se logre incorporar de manera efectiva o que no responda a las necesidades de los usuarios. La presencia de los usuarios meta es importante para garantizar que sus necesidades se conozcan, a fin de que la selección final de las TIC se dé en función de la satisfacción de esas necesidades.

\subsection{El nivel docente}

De acuerdo con el modelo de innovación de Fullan, las estrategias de innovación requieren de una combinación de cambios de arriba hacia abajo y de abajo hacia arriba. Según este modelo y, de acuerdo con la estrategia que aquí se plantea, es necesario implementar una combinación de cambios en el nivel institucional administrativo que tengan efecto sobre la docencia desde arriba hacia abajo y se debe introducir cambios en el área docente que produzcan un efecto sobre la administración desde abajo hacia arriba. Desde este punto de vista, los cambios en el nivel docente deben desarrollar las siguientes acciones: inserción y participación en los procesos de incorporación de las TIC; apoyo a las iniciativas de incorporación de las TIC y difusión de información relacionada con las TIC.

\subsubsection{Inserción y participación activa en el discurso de la incorporación de las TIC}

Los docentes deben insertarse y participar en las discusiones relacionadas con la incorporación de las TIC en la docencia pues esta estrategia les permitirá contribuir con sus argumentos e ideas a la solución de la problemática que aquí se discute.

Según Van Dijk (1993), el poder es un factor determinante en la conformación del discurso. Son los grupos socialmente poderosos los que determinan los niveles de acceso al discurso y los contextos en que éste se desarrolla. En el contexto universitario, los docentes conforman grupos de poder en asambleas de escuelas o facultades, comisiones 
universitarias y otras instancias de directa relación con la administración institucional. En este sentido, los docentes deben aprovechar su presencia en estos grupos poderosos para insertarse activamente en el discurso de la incorporación de las TIC en la docencia. Solo mediante el ejercicio de una participación visible y sonora en los contextos en los que se desarrolla este discurso, los docentes pueden dar a conocer, de manera efectiva, sus necesidades y las de sus estudiantes en el campo de las TIC y abogar para que se tomen decisiones que las satisfagan.

Este tipo de participación activa, visible y sonora ayudará a que se tenga una mejor perspectiva del impacto que tendrían las decisiones de incorporación de las TIC en la docencia y el aprendizaje. Aunque la participación de cada docente será diferente de acuerdo con el contexto en que se encuentra, él o ella debe asegurarse de reclamar su acceso y participación en este discurso para que el mismo tenga una mayor diversidad de argumentos en qué sustentar decisiones.

\subsubsection{Apoyo a las iniciativas de incorporación de las TIC}

Las iniciativas de incorporación de las TIC en la docencia requieren del apoyo de los docentes. Este apoyo debe ser concreto y visible. En otras palabras, los docentes deben hacer esfuerzos por adoptar las innovaciones que ofrezca la universidad. Al adoptar estas innovaciones, los docentes adquieren los insumos necesarios para evaluar el rendimiento y la efectividad de los procesos de incorporación de las TIC, y esta información se convierte en parte integral del discurso relacionado con estos procesos de incorporación. Si los docentes no incorporan y utilizan las TIC en su práctica profesional muy difícilmente ellos tendrían argumentos de peso para contribuir a este discurso.

\subsubsection{Difusión}

Tanto la participación en el discurso como el apoyo concreto a las iniciativas de incorporación deben estar acompañados por estrategias de difusión de parte de los docentes. Son ellos los que deben dar a conocer el estado de los procesos de incorporación de las TIC, la necesidad de actualizar los equipos e instrumentos, la efectividad de los programas de capacitación y las carencias adicionales que ellos, como usuarios, experimentan. Asímismo, los resultados positivos deben ser comunicados a otros docentes para estimular su participación. El conocimiento de los alcances y logros en la incorporación de las TIC en la docencia puede ser una fuente de motivación para aquellos que estén contemplando el participar en este proceso. 
Sin embargo, un aspecto fundamental que debe sustentar los esfuerzos de divulgación es la investigación. Es necesario que se realicen más investigaciones que den a conocer el impacto de las iniciativas de incorporación de las TIC y sus resultados en la docencia y en el aprendizaje. La investigación es importante para dotar al discurso de la incorporación de las TIC en la docencia de argumentos con sustento objetivo y científico.

\section{Conclusiones}

La implementación efectiva de las estrategias aquí planteadas puede tener efectos muy beneficiosos sobre la docencia entre las cuales se pueden destacar:

\subsection{Cambio de actitud}

Cuando la incorporación de las TIC responde a una visión institucional concreta y articulada se produce un efecto positivo sobre la cultura tecnológica de la universidad, ya que fomenta entre los docentes la reflexión sobre su papel y su experiencia en el proceso de incorporación de las TIC en la docencia; y para los docentes que aún no lo han hecho, los confronta con la necesidad de tomar una decisión sobre su propia inserción en estos procesos.

\subsection{Mayor espacio discursivo a la incorporación de las TIC}

La existencia de una política institucional de incorporación de las TIC en la docencia daría un mayor espacio y sonoridad a esta temática en el gran discurso institucional universitario pues en estos niveles es más factible que todas las partes interesadas sean escuchadas por la colectividad. Al ampliarse la participación en este discurso se abren más oportunidades para difundir información sobre los resultados positivos de la incorporación de las TIC en la docencia. Si los docentes tienen más acceso a información sobre estos resultados positivos, podrían estar más interesados en incorporar las TIC en la docencia, ya que generalmente los usuarios están más dispuestos a adoptar una innovación tecnológica si su percepción de estas innovaciones es positiva (Farquar y Surrey, 1994).

\subsection{Motivación}

La implementación de una estrategia institucional de incorporación de las TIC en la docencia definitivamente motivaría a más profesores a utilizarlas o, al menos, considerarlas. De hecho, estudios como los de Schifter demuestran que factores externos como el apoyo institucional son elementos importantes para motivar a los profesores a adoptar TIC en la docencia. 


\subsection{Beneficio para las universidades}

Para las universidades estatales el beneficio de la incorporación de las TIC sería enorme pues, entre otros aspectos, permitiría a las unidades académicas estar mejor preparadas para asumir los retos y las exigencias que, en esta materia, tienen las agencias evaluadoras y acreditadoras. Además, la implementación de estrategias de incorporación de las TIC redundaría en mayores niveles de competitividad y prestigio para las universidades.

\section{Reflexión final}

La problemática fundamental de la incorporación de las TIC en la docencia reside en un cambio de actitud de parte de las autoridades universitarias. Sin embargo, para efectuar este cambio los insumos principales son la voluntad y la buena disposición de las autoridades para tomar las acciones necesarias de forma estratégica y ordenada, y la participación activa y visible de los docentes. Al final, la solución de la problemática dependerá de que las instituciones reconozcan la necesidad de un esfuerzo conjunto de parte de las autoridades, los docentes y los estudiantes para asegurar el éxito de las estrategias que permitan una incorporación eficaz de las TIC en la docencia universitaria estatal costarricense.

\section{Referencias}

Adams, N. B. (2002). Educational computing concerns of postsecondary faculty. Journal of Research on Technology in Education. 34 (3): 285-303.

Bates, A. W. (2001). Cómo gestionar el cambio tecnológico. Barcelona: Gedisa.

Bates, A. W. (2000). Managing technological change: strategies for college and university leaders. San Francisco: Jossey-Bass.

Cuban, L. (2001). Oversold and underused. Computers in the classroom. Cambridge: Harvard University Press.

Epper, R. (2004). La torre de marfil de la nueva economía. En Enseñar al Profesorado Cómo Utilizar la Tecnología: Buenas prácticas de instituciones líderes. Barcelona: Editorial UOC. 11-31.

Ehrman, S. C. (1995). What does research tell us about technology and higher learning? Change. 27 (2): 20-27.

Escamez, J. y Martínez, F. (1987). Actitudes de los agentes educativos ante la informática. En Educar para el Siglo XXI. Madrid: Fundesco. 79-126. 
Fullan, M. (1994). Coordinating top-down and bottom-up strategies for educational reform. En The Governance of Curriculum. Alexandria: Association for Supervision and Curriculum Development. 186-202.

Green, K. C. (2003). Campus computing 2002: the 13th national survey of computing and information technology in american higher education. Encino: The Campus Computing Project.

Hoffman, R. (1996). School technology integration: An automated needs assessment and planning tool. En Technology and Teacher Education Annual, 1996. Charlottsville: Association for the Advancement of Computing in Education.

Farquhar, J. D. y Surry, D. W. (1994). Adoption analysis: an additional tool for instructional developers. Education and Training Technology International. 31 (1): 19-25.

Jonassen, D.; Chad C. y Yueh, H. (1998). Computers as mindtools for engaging learners in critical thinking. TechTrends. 43 (2): 24-32.

Kagima, L.K. y Hausafu, C. O. (2001). Faculty: The central element in instructional technology integration. Journal of Family and Consumer Science. 93 (4): 33-36.

Latona, K. (1996). Case studies in flexible learning. Sydney: University of Technology.

Lee, C.Y. (2000). Student motivation in an online learning environment. Journal of Educational Media and Library Sciences. 37 (4): 367-375.

Leggert, W. P. y Persichitte, K. A. (1998). Blood, sweat and TEARS: 50 years of technology implementation obstacles. TechTrends. 43 (3): 33-36.

Pasquinelli, A. (ed). (1998). Higher education and information technology: Trends and issues. Palo Alto: SUN Microsystems. Recuperado el 22 de setiembre de 2004 de http://www.sun.com/products-n-solutions/edu/admin/janeu2.pdf

Moran, L. y Myringer, B. (1999). Flexible learning and university change. En Higher Education Through Open and Distance Learning. Londres: Routeledge. 57-72.

Rao, P.V., y Rao, L. M. (1999, Marzo). Strategies that support instructional technology. Syllabus. (12): 22-24.

Rockwell, K., Schauer, J., Fritz, S. M., y Marx, D. B. (1999). Incentives and obstacles influencing higher education faculty and administrators to teach via distance. Online Journal of Distance Learning Administration. 2 (3). Recuperado el 15 de abril de 2005 de http://www.westga.edu/ distance/rockwell24.html

Rogers, D. (2000). A Paradigm Shift: technology integration for higher education in the new millenium. Educational Technology Review. 6 (3): 19-27.

Rogers, E. M. (1986). Communication technology: the new media in society. New York: Free Press.

Salinas, Jesús (2004). Innovación docente y uso de las TIC en la enseñanza universitaria. Revista de Universidad y Sociedad del Conocimiento. 1 (1). Fecha de publicación noviembre de 2004 en http://www.uoc.edu/rusc/dt/esp/salinas1104.pdf 
Salinas, Jesús (2002, Enero-Junio). Modelos flexibles como respuesta de las universidades a la sociedad de información. Acción Pedagógica. 11 (1): 4-13.

Salinas, Jesús. (1997). Enseñanza flexible, aprendizaje abierto. Las redes como herramientas para la formación. En Recursos Tecnológicos para los Procesos de Enseñanza y Aprendizaje. Málaga: ICE/Universidad de Málaga.

Schifter, Catherine. (2000, Junio). Faculty Participation in Asynchronous Learning Networks: A Case Study of Motivating and Inhibiting Factors. Journal of Asynchronous Learning. 4 (1): 15-22.

Schramm, W. (1977). Big Media, little media: tools and technologies for instruction. Beverly Hills: Sage.

Sherry, Lorraine. (1998). An Integrated adoption and diffusion model. International Journal of Educational Telecommunications. 4 (2/3): 113-145.

Stockdill, S. H., y Morehouse, D.L. (1992). Critical factors in the successful adoption of technology: A checklist based on TDC findings. Educational Technology. 1, 57-58.

Trevino, L. K., Lengel, R. H. y Daft, R. L. (1987). Media symbolism, media richness, and media choice in organizations: a symbolic interactionist perspective. Communication Research. 14 (5): 553-74.

Van Dijk, T. A. 1993. Principles of critical analysis. Discourse \& Society. 4 (2): 249-283.

Van Dusen, G. C. Technology: Higher Education's Magic bullet. The NEA Higher Education Journal. Recuperado el 03 de mayo de 2005 de http://www2.nea.org/he/heta98/s98pg59.pdf

Van Gelder, Tim. (2005). Teaching Critical Thinking. Some Lessons from Cognitive Science. College Teaching. 45:1-6. 